\title{
APPROXIMATION OF SOME DIFFUSION EVOLUTION EQUATIONS IN UNBOUNDED DOMAINS BY HERMITE FUNCTIONS
}

\author{
DANIELE FUNARO AND OTARED KAVIAN
}

\begin{abstract}
Spectral and pseudospectral approximations of the heat equation are analyzed. The solution is represented in a suitabie basis constructed with Hermite polynomials. Stability and convergence estimates are given and numerical tests are discussed.
\end{abstract}

\section{INTRODUCTION}

Many physical models involve the determination of the solution of a partial differential equation in an unbounded domain. The conditions at infinity are in general given by a certain asymptotic behavior for the solution. This could be obtained, for instance, by requiring a prescribed rate of decay at infinity. From the point of view of numerical approximation, it is not an easy task to give a constructive interpretation of the behavior at infinity. Among the techniques, one of the most widely used is to restrict the computation to a finite domain and impose some relations on the "artificial boundary" according to the physics of the problem.

As to the approximation by spectral methods, the literature for this kind of problems to our knowledge is quite sparse. We may quote three papers. The first by C. Canuto, S. I. Hariharan, and L. Lustman [1] deals with the approximation of an exterior elliptic problem in two dimensions by imposing an appropriate farfield condition at the artificial boundary in order to recover spectral convergence. In the second by Y. Maday, B. Pernaud-Thomas, and H. Vandeven [13], the solution of

$$
u_{t}+u_{x}=0, \quad(t, x) \in(0, T) \times(0,+\infty),
$$

is approximated by a truncated series of Laguerre polynomials. Finally, $\mathrm{O}$. Coulaud, D. Funaro, and O. Kavian [3] consider the numerical approximation of the solution to

$$
-\Delta u+\lambda u=f,
$$

in the exterior of a ball or a square, using Laguerre polynomials.

Received July 6, 1988; revised October 25, 1990.

1980 Mathematics Subject Classification (1985 Revision). Primary 35K05, 42C10, 65N35.

Key words and phrases. Parabolic equations, spectral approximation, Hermite polynomials, unbounded domains. 
In this paper we are concerned with the approximation of parabolic (or elliptic) problems by expanding the solution in the basis of the Hermite functions, i.e., Hermite polynomials multiplied by a Gaussian. This kind of approach is suggested when the decay at infinity is at least like $\exp \left(-\alpha x^{2}\right)$ for some $\alpha>0$. For a certain class of evolution equation, when the initial data satisfies the required decay condition, one can prove that the solution has at each time the same decay. As we show in $\S 1$, Hermite functions are a very natural choice for the approximation of solutions to diffusion PDE's, after an appropriate change of the space and time variables (the so-called similarity transformation). In $\S 2$, we study the spectral Galerkin approximation, we prove the convergence of the scheme and we give error estimates. Section 3 is devoted to the analysis of the pseudospectral approximation for the case in which the domain is $\mathbb{R}^{d}, d \geq 1$. Collocation is imposed at the zeros of the Hermite polynomial of degree $N+1$. We note that, since the zeros of Hermite polynomials spread all over the infinite domain with increasing $N$, no restriction in the size of the domain of approximation is required, and an artificial boundary does not exist. In $\S 4$ the results are generalized to cover the case of problems in unbounded domains which are Cartesian products of intervals; the case of approximation on a half straight line is also investigated. Finally, in $\S 5$ we give and discuss several numerical examples in one and two dimensions. Also we briefly indicate how to adapt the previous analysis to other situations.

\section{StATEMENT OF THE PROBlem}

In the study of qualitative properties and the numerical approximation of solutions to equations such as:

$$
\left\{\begin{array}{l}
u_{t}-\Delta u+F(u)=0 \text { in } \Omega, \\
u(0, x)=u_{0}(x), \\
u(t, \cdot) \text { satisfies certain boundary conditions on } \partial \Omega,
\end{array}\right.
$$

a crucial role is played by the fact that the operator $A:=-\Delta$ associated with the specified boundary conditions on the space $L^{2}(\Omega)$ has, or does not have, a compact resolvent. For instance, if the prescribed boundary condition is the homogeneous Dirichlet condition $u(t, \cdot)=0$ on $\partial \Omega$, and the domain $\Omega$ is bounded with $\partial \Omega$ Lipschitzian, then the domain of $A$ is contained in $H_{0}^{1}(\Omega)$, and by Rellich's theorem the resolvent of $A$ is compact. Now, as is well known, for the numerical approximation of the solution by a spectral, pseudospectral, or a finite element method, the compactness assumption is particularly important.

When $\Omega$ is unbounded, say $\Omega=\mathbb{R}^{d}$ or $\Omega=\mathbb{R}^{d-1} \times \mathbb{R}_{+}$, the operator $A$ no longer has a compact resolvent. In this case, as we shall see in the sequel, one may use the whole structure of the linear operator $\partial_{t}-\Delta$. In order to make this idea more transparent, we consider as an example the following Stokes 
evolution equation:

$$
\left\{\begin{array}{l}
u_{t}-\Delta u+\nabla p=f, \quad t>0, x \in \mathbb{R}^{d}, \\
u(0, x)=u_{0}(x), \\
\nabla \cdot u(t, x)=0
\end{array}\right.
$$

where $u=\left(u_{1}, \ldots, u_{d}\right) \in \mathbb{R}^{d}, d=2$ or 3 , and $f$ and $u_{0}$ are given in suitable spaces. Here, since the domain is $\mathbb{R}^{d}$, the inclusion $H^{1}\left(\mathbb{R}^{d}\right) \subset L^{2}\left(\mathbb{R}^{d}\right)$ is not compact. As suggested in M. Escobedo and O. Kavian [6] and O. Kavian [9] in the study of the parabolic equations

$$
u_{t}-\Delta u \pm|u|^{p-1} u=0,
$$

one can associate with the function $u$ and the operator $\left(\partial_{t}-\Delta\right)$ another function $v$ and another operator $\left(\partial_{t}+L\right)$ such that the new elliptic operator $L$ (in an appropriate Hilbert space) has a compact resolvent. Moreover, the functions $u$ and $v$ are related in a simple way. More precisely, if $u$ and $p$ are given by (1.2), define $v, q$, and $g$ by

$$
\begin{aligned}
& u(t, x)=: v\left(\log (1+t), \frac{x}{\sqrt{1+t}}\right), \\
& p(t, x)=:(1+t)^{-1 / 2} q\left(\log (1+t), \frac{x}{\sqrt{1+t}}\right), \\
& f(t, x)=:(1+t)^{-1} g\left(\log (1+t), \frac{x}{\sqrt{1+t}}\right) .
\end{aligned}
$$

Now setting

$$
s:=\log (1+t), \quad y:=\frac{x}{\sqrt{1+t}}
$$

one checks easily that $(v, q)$ satisfies

$$
\left\{\begin{array}{l}
v_{s}+L v+\nabla q=g, \quad s>0, y \in \mathbb{R}^{d}, \\
v(0, y)=u_{0}(y), \\
\nabla \cdot v(s, y)=0,
\end{array}\right.
$$

where in the first equation of (1.5), instead of the elliptic operator $-\Delta$, we have the elliptic operator $L$ defined by

$$
L \varphi:=-\Delta \varphi-\frac{y \cdot \nabla \varphi}{2} .
$$

By the results in [5] this operator is selfadjoint in the weighted Lebesgue space:

$$
L_{w}^{2}\left(\mathbb{R}^{d}\right):=\left\{f ; \int_{R^{d}}|f(y)|^{2} w(y) d y<\infty\right\},
$$

where $w(y):=\exp \left(|y|^{2} / 4\right)$, and has a compact inverse. Furthermore, in the special case considered here, i.e., $\Omega=\mathbb{R}^{d}$, the eigenvalues and eigenfunctions of $L$ are known, namely (we denote here, for $\alpha \in \mathbb{N}^{d},|\alpha|:=\alpha_{1}+\cdots+\alpha_{d}$ ):

$$
L \varphi_{j, \alpha}=\lambda_{j} \varphi_{j, \alpha},
$$


where the $j$ th eigenvalue $\lambda_{j}$ is

$$
\lambda_{j}:=\frac{d+j-1}{2} \text { for } j \geq 1,
$$

and the corresponding eigenfunctions are

$$
\begin{aligned}
\varphi_{j, \alpha}(y):=(-1)^{|\alpha|} D^{\alpha}\left(\exp \left(\frac{-|y|^{2}}{4}\right)\right) & \\
& \alpha \in \mathbb{N}^{d},|\alpha|=j-1, D^{\alpha}:=\frac{\partial^{\alpha_{1}}}{\partial y_{1}^{\alpha_{1}}} \cdots \frac{\partial^{\alpha_{d}}}{\partial y_{d}^{\alpha_{d}}} .
\end{aligned}
$$

These eigenfunctions are nothing but the Hermite functions, and can be written in terms of Hermite polynomials. We define these by

$$
h_{m}(x):=(-1)^{m} \exp \left(\frac{x^{2}}{4}\right) \frac{d^{m}}{d x^{m}}\left(\exp \left(\frac{-x^{2}}{4}\right)\right)
$$

for $m \in \mathbb{N}$ and $x \in \mathbb{R}$.

Then setting

$$
H_{\alpha}(y):=h_{\alpha_{1}}\left(y_{1}\right) \cdots h_{\alpha_{d}}\left(y_{d}\right)
$$

we have

$$
\begin{aligned}
\varphi_{j, \alpha}(y) & =\prod_{1 \leq i \leq d} h_{\alpha_{i}}\left(y_{i}\right) \exp \left(\frac{-y_{i}^{2}}{4}\right)=H_{\alpha}(y) \exp \left(\frac{-|y|^{2}}{4}\right), \\
y & =\left(y_{1}, \ldots, y_{d}\right) \in \mathbb{R}^{d}, \alpha=\left(\alpha_{1}, \ldots, \alpha_{d}\right) \in \mathbb{N}^{d},|\alpha|=j-1 .
\end{aligned}
$$

Note that definition (1.10) differs slightly from the one used in the literature where, instead of $\exp \left(-x^{2} / 4\right)$, one has $\exp \left(-x^{2} / 2\right)$ or $\exp \left(-x^{2}\right)$. Now the resolution of the elliptic equation

$$
L \psi=f, \quad f \in L_{w}^{2}\left(\mathbb{R}^{d}\right),
$$

or of the parabolic equation

$$
\left\{\begin{array}{l}
\partial_{s} \varphi+L \varphi=0 \\
\varphi(0, \cdot)=f(\cdot) \in L_{w}^{2}\left(\mathbb{R}^{d}\right)
\end{array}\right.
$$

in terms of the above eigenfunctions is quite simple. Indeed (here $\alpha$ ! := $\left.\prod_{1 \leq i \leq d}\left(\alpha_{i} !\right)\right)$ :

$$
\psi(y)=\sum_{\alpha \in N^{d}} \frac{2}{|\alpha|+d} c_{\alpha} H_{\alpha}(y) e^{-|y|^{2} / 4}
$$

and

$$
\varphi(s, y)=\sum_{\alpha \in \mathbb{N}^{d}} c_{\alpha} \exp \left(-\lambda_{1+|\alpha|} s\right) H_{\alpha}(y) \exp \left(-|y|^{2} / 4\right)
$$


where the coefficients $c_{\alpha}$ are

$$
c_{\alpha}=\frac{\int_{R^{d}} f(y) H_{\alpha}(y) d y}{\int_{R^{d}}\left|H_{\alpha}(y)\right|^{2} \exp \left(-|y|^{2} / 4\right) d y}=\frac{2^{|\alpha|-1}}{\pi^{d / 2} \alpha !} \int_{R^{d}} f(y) H_{\alpha}(y) d y .
$$

This suggests, as we shall develop in the next sections, a numerical approximation of $\psi$ or $\varphi$ using a truncation of the expansions (1.15) or (1.16).

Remark 1.1. The so-called similarity transformation (1.3) introduced for the study of the linear system (1.2) can be modified to be applied to the nonlinear Navier-Stokes equation. Indeed, if $(u, p)$ is a solution to

$$
u_{t}-\Delta u+(u \cdot \nabla) u+\nabla p=f, \quad \nabla \cdot u=0,
$$

then we define $(v, q)$ and $g$ by

$$
\begin{aligned}
& u(t, x)=:(1+t)^{-1 / 2} v\left(\log (1+t), \frac{x}{\sqrt{1+t}}\right), \\
& p(t, x)=:(1+t)^{-1} q\left(\log (1+t), \frac{x}{\sqrt{1+t}}\right), \\
& f(t, x)=:(1+t)^{-3 / 2} g\left(\log (1+t), \frac{x}{\sqrt{1+t}}\right) .
\end{aligned}
$$

Setting $s$ and $y$ as in (1.4), one checks easily that $(v, q)$ satisfies

$$
v_{s}+L v+(v \cdot \nabla) v+\nabla q-\frac{1}{2} v=g, \quad \nabla \cdot v=0
$$

where $L$ is the operator defined in (1.6). In [10] this equation is studied in the space $L_{w}^{2}\left(\mathbb{R}^{d}\right)$.

Remark 1.2. For $f \equiv 0, \mathrm{~J}$. Leray in [11] seeks a self-similar solution $u$ to (1.18) (which blows up at time, say, $T:=1$ ) by setting

$$
u(t, x)=(1-t)^{-1 / 2} Z\left(\frac{x}{\sqrt{1-t}}\right), \quad 0 \leq t<1 .
$$

It turns out that such a function $Z$ would satisfy

$$
-\Delta Z+\frac{y \cdot \nabla Z}{2}+(Z \cdot \nabla) Z+\nabla q+\frac{1}{2} Z=0, \quad \nabla \cdot Z=0 .
$$

Actually, one can prove that if $d=2$, and if $Z \in H^{1}\left(\mathbb{R}^{2}\right)$ satisfies (1.21), then $Z \equiv 0$ (cf. [10]). For $d=3$, as far as we know, it is an open question whether (1.21) has a nontrivial solution or not. On the other hand, one can look for self-similar solutions of (1.18) which are global in time, i.e., such that

$$
u(t, x)=t^{-1 / 2} \widetilde{Z}\left(\frac{x}{\sqrt{t}}\right), \quad t>0,
$$

i.e., $\tilde{Z}$ would be a steady state for the evolution equation (1.20). When $\tilde{Z} \in$ $H^{1}\left(\mathbb{R}^{d}\right)$ and $d \geq 2$, one can prove that $\tilde{Z} \equiv 0$.

\section{Galerkin APPROXIMATION}

For the reader's convenience we begin by recalling some well-known properties of Hermite polynomials. Let $a>0$ be a fixed parameter. For $n \in \mathbb{N}$ we 
define the $n$th Hermite polynomial on $\mathbb{R}$ by

$$
h_{n, a}(x):=(-1)^{n} e^{x^{2} / 4 a^{2}} \frac{d^{n}}{d x^{n}}\left(e^{-x^{2} / 4 a^{2}}\right) .
$$

We shall denote as in (1.10) $h_{n}:=h_{n, 1}$. These polynomials have the following properties (cf. for instance [4, 14]):

$$
2 a^{2} h_{n, a}^{\prime \prime}+n h_{n, a}=x h_{n, a}^{\prime},
$$

$$
\left\{\begin{array}{l}
h_{0, a}(x)=1, \\
h_{1, a}(x)=x / 2 a^{2}, \\
4 a^{2} h_{n, a}(x)=2 x h_{n-1, a}(x)-2(n-1) h_{n-2, a}(x) \text { for } n \geq 2,
\end{array}\right.
$$

and satisfy the orthogonality relations

$$
\int_{\mathbb{R}} h_{n, a}(x) h_{m, a}(x) e^{-x^{2} / 4 a^{2}} d x=2^{1-n} a^{1-2 n} n ! \sqrt{\pi} \delta_{n}^{m} \text { for } n, m \geq 0 .
$$

Our aim is to approximate the solution of the following parabolic equation $(u(t, x) \in \mathbb{R})$ :

$$
\begin{cases}\partial_{t} u-a^{2} \Delta u=f, & t<0, x \in \Omega, \\ u(0, x)=u_{0}(x), & x \in \Omega, \\ u(t, \cdot)_{\mid \partial \Omega}=0, & t>0, \\ \Omega=\mathbb{R}^{d} \text { or } \mathbb{R}^{d-1} \times(0, \infty) .\end{cases}
$$

Defining $v$ and $g$ as in (1.3), and $s$ and $y$ as in (1.4), one checks that

$$
\begin{cases}\partial_{s} v-a^{2} \Delta v-y \cdot \nabla v / 2=g, & s>0, y \in \Omega, \\ v(0, y)=u_{0}(y), & y \in \Omega, \\ v(s, \cdot)_{\mid \partial \Omega}=0, & s>0 .\end{cases}
$$

In the sequel we shall use the following notations and functional spaces:

$$
\begin{gathered}
w_{a}(y):=\exp \left(\frac{|y|^{2}}{4 a^{2}}\right), \\
L_{a} \varphi:=-a^{2} \Delta \varphi-\frac{y \cdot \nabla \varphi}{2}=-\frac{a^{2}}{w_{a}} \nabla \cdot\left(w_{a} \nabla \varphi\right), \\
\text { (2.8) } \quad L_{w_{a}}^{2}(\Omega):=\left\{\varphi: \Omega \rightarrow \mathbb{R} \text { measurable; } \int_{\Omega}|\varphi(y)|^{2} w_{a}(y) d y<\infty\right\} .
\end{gathered}
$$

For integers $m \geq 1$,

$$
\begin{gathered}
H_{w_{a}}^{m}(\Omega):=\left\{\varphi \in L_{w_{a}}^{2}(\Omega) ; D^{\alpha} \varphi \in L_{w_{a}}^{2}(\Omega),|\alpha| \leq m\right\}, \\
\text { (2.11) } \quad H_{w_{a}, 0}^{m}(\Omega):=\left\{\varphi \in H_{w_{a}}^{m}(\Omega) ; D^{\alpha} \varphi=0 \text { on } \partial \Omega, \text { for }|\alpha| \leq m-1\right\} .
\end{gathered}
$$


On $L_{w_{a}}^{2}(\Omega)$ we denote the scalar product and norm by

$$
\begin{gathered}
(\varphi \mid \psi)_{0, a}:=\int_{\Omega} \varphi(y) \psi(y) w_{a}(y) d y, \\
\|\varphi\|_{0, a}:=(\varphi \mid \varphi)_{0, a}^{1 / 2},
\end{gathered}
$$

and for $m \geq 1$ and $\varphi, \psi \in H_{w_{a}}^{m}(\Omega)$ :

$$
\begin{gathered}
(\varphi \mid \psi)_{m, a}:=\sum_{|\alpha| \leq m}\left(D^{\alpha} \varphi \mid D^{\alpha} \psi\right)_{0, a}, \\
\|\varphi\|_{m, a}:=(\varphi \mid \varphi)_{m, a}^{1 / 2} .
\end{gathered}
$$

For $0<\tau<1$, we introduce the interpolation spaces

$$
H_{w_{a}}^{m+\tau}(\Omega):=\left[H_{w_{a}}^{m}(\Omega), H_{w_{a}}^{m+1}(\Omega)\right]_{1-\tau}
$$

in the sense of [12]. When $a=1$ and $\Omega=\mathbb{R}^{d}$, it has been proved in [6, $\S \S 1$ and 2; 9, Lemma (2.1)] that $L_{1}$ is a selfadjoint operator acting on $L_{w_{1}}^{2}\left(\mathbb{R}^{d}\right)$ and that its inverse $L_{1}^{-1}$ exists and is compact. Using these results and a change of scale in the variable $y$, one can easily prove the following.

Lemma 2.1. Let $\Omega$ be as in (2.5) and set $D\left(L_{a}\right):=H_{w_{a}}^{2}(\Omega) \cap H_{w_{a}, 0}^{1}(\Omega)$. Then:

(i) $L_{a}$ is a selfadjoint operator on $L_{w_{a}}^{2}(\Omega)$ whose domain is $D\left(L_{a}\right)$.

(ii) $\forall \varphi \in H_{w_{a}}^{1}(\Omega), \frac{d}{2}|\varphi|_{0, a}^{2} \leq|\nabla \varphi|_{0, a}^{2}=\left(L_{a} \varphi \mid \varphi\right)_{0, a}$.

(iii) $L_{a}^{-1}$ exists and is compact on $L_{w_{a}}^{2}(\Omega)$.

Lemma 2.2. Let $\Omega$ be as in (2.5).

(i) The embedding $H_{w_{a}}^{m-1}(\Omega) \subset H_{w_{a}}^{m}(\Omega)$ is compact.

(ii) $\varphi \in H_{w_{a}}^{1}(\Omega) \Leftrightarrow w_{a}^{1 / 2} \varphi=w_{a \sqrt{2}} \varphi \in H^{1}(\Omega)$ and $|\cdot| w_{a}^{1 / 2} \varphi \in L_{w_{a}}^{2}(\Omega)$.

(Here, $H^{1}(\Omega)$ denotes the classical Sobolev space, and $|\cdot|$ is the function $y \mapsto$ $|y|$.

The eigenvalues and eigenfunctions of $L_{a}$ on $D\left(L_{a}\right)$ can be written explicitly in some cases. We denote by $\operatorname{Sp}\left(L_{a}\right):=\left\{\lambda_{j} ; j \geq 1\right\}$ the spectrum of $L_{a}$ on $D\left(L_{a}\right)$, and by $\varphi_{j}$ the normalized (in $L_{w}^{2}$ ) eigenfunctions corresponding to $\lambda_{j}$.

Proposition 2.3. Let $d=1$.

(i) If $\Omega=\mathbb{R}$, for $j \geq 1$ each eigenvalue $\lambda_{j}$ is simple, $\lambda_{j}=j / 2$, and the corresponding normalized eigenfunction $\varphi_{j}$ is given by

$$
\varphi_{j}(y):=\left(\frac{2^{j-2} a^{2 j-3}}{\sqrt{\pi}(j-1) !}\right)^{1 / 2} h_{j-1, a}(y) \exp \left(\frac{-|y|^{2}}{4 a^{2}}\right) .
$$


(ii) If $\Omega=(0, \infty)$, for $j \geq 1$ each eigenvalue $\lambda_{j}$ is simple, $\lambda_{j}=j$, and the corresponding normalized eigenfunction $\varphi_{j}$ is given by

$$
\varphi_{j}(y):=2\left(\frac{2^{2 j-2} a^{4 j-3}}{\sqrt{\pi}(2 j-1) !}\right)^{1 / 2} h_{2 j-1, a}(y) \exp \left(\frac{-|y|^{2}}{4 a^{2}}\right) .
$$

Proposition 2.4. Let $d \geq 2$ and $\Omega=\mathbb{R}^{d}$. For $\alpha=\left(\alpha_{1}, \ldots, \alpha_{d}\right) \in \mathbb{N}^{d},|\alpha|=$ $j-1 \geq 0$, the jth eigenvalue is $\lambda_{j}:=\lambda_{j, \alpha}:=(|\alpha|+d) / 2=(j+d-1) / 2$, with multiplicity $\left(\begin{array}{c}j+d-1 \\ j\end{array}\right)$. The corresponding normalized eigenfunctions are

$$
\varphi_{j, \alpha}(y):=\prod_{1 \leq i \leq d}\left(\frac{2^{\alpha_{i}-1} a^{2 \alpha_{i}-1}}{\sqrt{\pi}\left(\alpha_{i}\right) !}\right)^{1 / 2} h_{\alpha_{i}, a}\left(y_{i}\right) \exp \left(\frac{-y_{i}^{2}}{4 a^{2}}\right) .
$$

For other domains such as $\mathbb{R}^{d-1} \times(0, \infty)$ or $((0, \infty))^{d}$ one can find the eigenvalues and eigenfunctions by a suitable combination of the above.

We are now in a position to consider the Galerkin approximation of equation (2.6). Let $N \geq 1$ be a fixed integer; define the subspace $X_{N}$ of $L_{w_{a}}^{2}(\Omega)$ by

$$
X_{N}:=\operatorname{span}\left\{\varphi_{i, \alpha} ; 1 \leq i \leq N, \alpha \in \mathbb{N}^{d},|\alpha| \leq i-1\right\} .
$$

Note that, if $\Omega=\mathbb{R}^{d}$, then the dimension of $X_{N}$ is

$$
\operatorname{dim}\left(X_{N}\right)=\sum_{i=1}^{N}\left(\begin{array}{c}
i+d-1 \\
i
\end{array}\right)=N^{d} .
$$

For instance, if $d=1$, i.e., $\Omega=\mathbb{R}$, by Proposition 2.3(i), an element of $X_{N}$ is a polynomial of degree at most $(N-1)$ multiplied by $\exp \left(-y^{2} / 4 a^{2}\right)$. If $\Omega=(0, \infty)$, by Proposition 2.3(ii), an element of $X_{N}$ is an odd polynomial of degree at most $(2 N-1)$ multiplied by $\exp \left(-y^{2} / 4 a^{2}\right)$.

We approximate the solution $v$ of (2.6) by $v_{N} \in X_{N}$, the unique solution to

$$
\left\{\begin{array}{l}
\frac{d}{d s}\left(v_{N}(s) \mid \varphi\right)_{0, a}+\left(L_{a} v_{N}(s) \mid \varphi\right)_{0, a}=(g(s) \mid \varphi)_{0, a}, \\
v_{N}(0)=u_{0 N},
\end{array} \forall \varphi \in X_{N}, \forall s>0,\right.
$$

where $u_{0 N} \in X_{N}$ is the $L_{w_{a}}^{2}$-projection of $u_{0}$. One has the following convergence result:

Proposition 2.5. Let $T>0$ be given, and suppose that

$$
g \in L^{2}\left(0, T ; H_{w_{a}}^{\sigma-1}(\Omega)\right), \quad u_{0} \in H_{w_{a}}^{\sigma}(\Omega) \cap H_{w_{a}, 0}^{1}(\Omega), \quad \sigma \geq 1 .
$$

Then there exists a unique solution $v \in C^{0}\left(0, T ; H_{w_{a}}^{\sigma}(\Omega) \cap H_{w_{a}, 0}^{1}(\Omega)\right)$ to (2.6). The solution $v_{N}$ is uniquely defined by (2.17), and the following error estimate holds:

$$
\left\{\begin{array}{l}
\text { for } 0 \leq \mu \leq \sigma, \sigma \geq 1, \text { and } 0 \leq s \leq T: \\
\left\|v(s)-v_{N}(s)\right\|_{\mu, a} \leq C \lambda_{N+1}^{(\mu-\sigma) / 2}\left(e^{-\lambda_{N+1} s}\left\|u_{0}\right\|_{\sigma, a}+\|g\|_{L^{2}\left(0, T, H_{w_{a}}^{\sigma-1}\right)}\right),
\end{array}\right.
$$

where $C$ is independent of $N, s$, and $T$. 
Note that by Propositions 2.3 and 2.4 one sees that $\lambda_{N}$ is proportional to $N$; therefore the error behaves like $N^{-(\sigma-\mu) / 2}$.

The proof of Proposition 2.5 is classical and can easily be done by writing the Hilbert series for $\left(v(s)-v_{N}(s)\right)$ in the basis $\left(\varphi_{j, \alpha}\right)_{|\alpha|=j-1, j \geq 1}$.

Now returning to the original equation (2.5), it is natural to define an approximation of $u$ by $u_{N}(t, x):=v_{N}(\log (1+t), x / \sqrt{1+t})$. As a result of the previous proposition we can state the following:

Proposition 2.6. Let $T>0$ and $v_{N}$ be given by (2.17). Define for $t \geq 0$ and $x \in \Omega$

$$
u_{N}(t, x):=v_{N}\left(\log (1+t), \frac{x}{\sqrt{1+t}}\right) .
$$

Then for $0 \leq \mu \leq \sigma, \sigma \geq 1$, there exists a constant $C>0$ independent of $N, t$, and $T$ such that for $f$ and $u_{0}$ satisfying

$$
f \in L^{2}\left(0, T ; H_{w_{a}}^{\sigma-1}(\Omega)\right), \quad u_{0} \in H_{w_{a}}^{\sigma}(\Omega) \cap H_{w_{a}, 0}^{1}(\Omega)
$$

we have for $0 \leq t \leq T$ and $u$ satisfying (2.5) that

$$
\left\|u(t)-u_{N}(t)\right\|_{H^{\mu}(\Omega)} \leq C N^{(\mu-\sigma) / 2}\left((1+t)^{-N / 2}\left\|u_{0}\right\|_{\sigma, a}+\|f\|_{L^{2}\left(0, T, H_{w_{a}}^{\sigma-1}(\Omega)\right)}\right) .
$$

\section{ApProximation by the COLlocation METHOD IN THE WHOLE SPACE}

In this section we analyze the pseudospectral approximation of equation (2.6) via the formulation (2.17). We begin with the case $d=1, \Omega=\mathbb{R}$ and the elliptic version of (2.6) and (2.17). Namely, for given $\lambda>0$ and $g \in H_{w_{a}}^{\sigma}(\mathbb{R})$, $\sigma \geq 1$, we consider

$$
\lambda v-a^{2} v_{y y}-\frac{y v_{y}}{2}=g \text { for } y \in \mathbb{R}, v \in H_{w_{a}}^{\sigma+2}(\mathbb{R})
$$

and its pseudospectral approximation in the space $X_{N+1}$ (defined in (2.16)); here, $\tilde{g}_{N}$ is a suitable projection of $g$ defined below in (3.5):

$$
\lambda\left(v_{N} \mid \varphi\right)_{0, a}+\left(L_{a} v_{N} \mid \varphi\right)_{0, a}=\left(\tilde{g}_{N} \mid \varphi\right)_{0, a}, \quad \forall \varphi \in X_{N+1}, \quad v_{N} \in X_{N+1} .
$$

We know that $v_{N}$ is uniquely determined and that there exists a polynomial $P_{N}$ of degree (at most) $N$ such that

$$
v_{N}(y)=P_{N}(y) \exp \left(-\frac{y^{2}}{4 a^{2}}\right) .
$$

Therefore, finding $v_{N}$ is equivalent to finding $P_{N}$ or its values at $(N+1)$ points, which we choose to be the zeros of the Hermite polynomial $h_{N+1, a}$ defined in (2.1). For the reader's convenience we recall some integration formulae (see, for instance, [4, Chapter 3], where the results are given for $a=\frac{1}{2} ;$ a change of 
scale in the variable yields the general result). Define $y_{k, N+1, a}$ and $\omega_{k, N+1, a}$ by

$$
\omega_{k, N+1, a}:=\frac{(N+1) !}{2^{N+1} a^{2 N+3}} \frac{\sqrt{\pi}}{\left(h_{N+2, a}\left(y_{k, N+1, a}\right)\right)^{2}}
$$

for $1 \leq k \leq N+1, h_{N+1, a}\left(y_{k, N+1, a}\right)=0$. When it is not ambiguous, we shall write $y_{k}, \omega_{k}$ instead of $y_{k, N+1, a}, \omega_{k, N+1, a}$.

The following integration formula holds for any polynomial $P$ of degree $\leq 2 N+1$ :

$$
\int_{-\infty}^{+\infty} P(y) \exp \left(-\frac{y^{2}}{4 a^{2}}\right) d y=\sum_{k=1}^{N+1} \omega_{k, N+1, a} P\left(y_{k, N+1, a}\right) .
$$

For a given function $g \in H_{w_{a}}^{\sigma}(\mathbb{R})$, with $\sigma>d / 2$, we define the pseudospectral projection of $g$ onto $X_{N+1}$ by

$$
\left\{\begin{array}{l}
p s_{N}(g):=\tilde{g}_{N}:=Q_{N} / w_{a} \in X_{N+1} \\
Q_{N} \text { is a polynomial, } \operatorname{deg} Q_{N} \leq N \\
Q_{N}\left(y_{k, N+1, a}\right)=w_{a}\left(y_{k, N+1, a}\right) g\left(y_{k, N+1, a}\right), \quad 1 \leq k \leq N+1 .
\end{array}\right.
$$

The collocation method to solve (3.2) is defined by

$$
\left\{\begin{array}{l}
\text { find a polynomial } P_{N}, \operatorname{deg} P_{N} \leq N, \text { such that for } 1 \leq i \leq N+1: \\
-a^{2} P_{N}^{\prime \prime}\left(y_{i}\right)+\frac{y_{i}}{2} P_{N}^{\prime}\left(y_{i}\right)+\frac{P_{N}\left(y_{i}\right)}{2}+\lambda P_{N}\left(y_{i}\right)=Q_{N}\left(y_{i}\right),
\end{array}\right.
$$

which is obtained from (3.2) by using relation (3.4), after $v_{N}$ is substituted by $\tilde{v}_{N}=P_{N} / w_{a}$. Introducing the Lagrange interpolation polynomials

$$
\ell_{j}(y):=\frac{h_{N+1, a}(y)}{h_{N+1, a}^{\prime}\left(y_{j}\right)\left(y-y_{j}\right)}, \quad 1 \leq j \leq N+1,
$$

one can write

$$
P_{N}(y)=\sum_{j=1}^{N+1} P_{N}\left(y_{j}\right) \ell_{j}(y)
$$

(In fact, $\ell_{j}$ depends on $N$ and $a$; but for simplicity we drop these indices as we do for $y_{j}$. ) An easy computation (using (2.2)) shows that

$$
\begin{aligned}
& \ell_{j}^{\prime}\left(y_{i}\right)= \begin{cases}\frac{h_{N+1, a}^{\prime}\left(y_{i}\right)}{h_{N+1, a}^{\prime}\left(y_{j}\right)} \frac{1}{\left(y_{i}-y_{j}\right)} & \text { if } i \neq j, \\
\frac{y_{i}}{4 a^{2}} & \text { if } i=j,\end{cases} \\
& \ell_{j}^{\prime \prime}\left(y_{i}\right)= \begin{cases}\frac{h_{N+1, a}^{\prime}\left(y_{i}\right)}{h_{N+1, a}^{\prime}\left(y_{j}\right)} \frac{1}{\left(y_{i}-y_{j}\right)^{2}}\left(\left(y_{i}-y_{j}\right) \frac{y_{i}}{2 a^{2}}-2\right) & \text { if } i \neq j, \\
\frac{1}{3 a^{2}}\left(\frac{y_{j}^{2}}{4 a^{2}}-\frac{N}{2}\right) & \text { if } i=j .\end{cases}
\end{aligned}
$$


Therefore, problem (3.6) can be written as a linear system in the Lagrange basis. Remark 3.1. The eigenvalues relative to the pseudospectral approximation of the operator $L_{a}$ are explicitly known. They are the same as in the Galerkin approximation, i.e.,

$$
\lambda_{j}=j / 2 \text { for } j=1, \ldots, N+1 \text {. }
$$

Let us note that they do not depend on $a$.

In the following we denote by $\hat{u}$ the Fourier transform of $u$, and by $\rho$ the function $\rho(y):=\left(1+|y|^{2}\right)^{1 / 2}$ defined on $\mathbb{R}^{d} \rightarrow \mathbb{R}_{+}$. By classical results and techniques in Sobolev spaces (see, for instance, [12]) one can state the following proposition (cf. [10] for a detailed proof):

Proposition 3.2. Let $d \geq 1$ be an integer and $\sigma \geq 0$ real. Define

$$
\mathscr{S}^{\sigma}\left(\mathbb{R}^{d}\right):=\left\{u ; u \in H^{\sigma}\left(\mathbb{R}^{d}\right) \text { and } \hat{u} \in H^{\sigma}\left(\mathbb{R}^{d}\right)\right\} .
$$

Then $\mathscr{S}^{\sigma}\left(\mathbb{R}^{d}\right)$, equipped with the norm

$$
\|u\|_{\mathscr{S}^{\sigma}}:=\left(\|u\|_{H^{\sigma}}^{2}+\|\hat{u}\|_{H^{\sigma}}^{2}\right)^{1 / 2}
$$

is a Hilbert space, and the following holds (we denote by $[\sigma]$ the integer part of $\sigma)$ :

(i) $u \in \mathscr{S}^{\sigma}\left(\mathbb{R}^{d}\right) \Leftrightarrow \rho^{\sigma} u \in L^{2}\left(\mathbb{R}^{d}\right)$ and $\rho^{\sigma} \hat{u} \in L^{2}\left(\mathbb{R}^{d}\right)$.

(ii) $u \in \mathscr{S}^{\sigma}\left(\mathbb{R}^{d}\right) \Leftrightarrow \forall \alpha \in \mathbb{N}^{d},|\alpha| \leq \sigma, \rho^{\sigma-\alpha} D^{\alpha} u \in L^{2}\left(\mathbb{R}^{d}\right)$, and $\rho^{\sigma-\alpha} D^{\alpha} \hat{u} \in$ $L^{2}\left(\mathbb{R}^{d}\right)$. Moreover, the norms are equivalent.

(iii) $u \in \mathscr{S}^{\sigma}\left(\mathbb{R}^{d}\right) \Leftrightarrow \forall \mu, 0 \leq \mu \leq \sigma, \rho^{\sigma-\mu}\left(\widehat{\rho^{\mu} u}\right) \in L^{2}\left(\mathbb{R}^{d}\right)$. Moreover, the norms are equivalent.

(iv) $u \in H_{w_{a}}^{\sigma} \Leftrightarrow w_{a}^{1 / 2} u \in \mathscr{S}^{\sigma}\left(\mathbb{R}^{d}\right)$. Moreover, the norms are equivalent.

Using this proposition, one may prove the following:

Proposition 3.3. Let $\sigma>d / 2$ and $0 \leq \mu<\sigma-d / 2$ be given. For any $u \in$ $\mathscr{S}^{\sigma}\left(\mathbb{R}^{d}\right)$ one has $\rho^{\mu} u \in C_{0}\left(\mathbb{R}^{d}\right)$ and there exists a constant $C$ (depending only on $\sigma, \mu$, and $d$ ) such that

$$
\left\|\rho^{\mu} u\right\|_{\infty} \leq C\|u\|_{\mathscr{S}^{\sigma}} .
$$

Proof. In order to see that $\rho^{\mu} u \in C_{0}\left(\mathbb{R}^{d}\right)$, it is sufficient to have $\left(\widehat{\rho^{\mu}} u\right) \in$ $L^{1}\left(\mathbb{R}^{d}\right)$. Indeed, $\forall x \in \mathbb{R}^{d}$,

$$
\begin{gathered}
\rho^{\mu}(x) u(x)=\int_{R^{d}} e^{2 i \pi x \cdot \xi}\left(\widehat{\rho^{\mu} u}\right)(\xi) d \xi \\
\left|\rho^{\mu}(x) u(x)\right| \leq\left\|\left(\widehat{\rho^{\mu} u}\right)\right\|_{L^{1}} \\
\leq\left(\int_{R^{d}}\left|\rho^{\sigma-\mu}(\xi)\left(\widehat{\rho^{\mu} u}\right)(\xi)\right|^{2} d \xi\right)^{1 / 2}\left(\int_{R^{d}}\left|\rho^{\sigma-\mu}(\xi)\right|^{-2} d \xi\right)^{1 / 2} \\
\leq C\|u\|_{\mathscr{S}^{\sigma}},
\end{gathered}
$$


where in the last inequality we use (iii) of Proposition 3.2, noting that $\int_{R^{d}}\left|\rho^{\sigma-\mu}(\xi)\right|^{-2} d \xi$ is finite for $2(\sigma-\mu)>d$.

Combining Proposition 3.3 and (iv) of Proposition 3.2 yields:

Corollary 3.4. Let $\sigma>d / 2$ and $0 \leq \mu<\sigma-d / 2$ be given. For any $u \in H_{w_{a}}^{\sigma}\left(\mathbb{R}^{d}\right)$ one has $\rho^{\mu} w_{a}^{1 / 2} u \in C_{0}\left(\mathbb{R}^{d}\right)$ and there exists a constant $C$ (depending only on $\sigma, \mu$, and $d$ ) such that

$$
\left\|\rho^{\mu} w_{a}^{1 / 2} u\right\|_{\infty} \leq C\|u\|_{\sigma, a} .
$$

Next we prove the convergence of $p s_{N}(g)$ to $g$ (in appropriate norms) as $N \rightarrow \infty$.

Theorem 3.5. Let $\varepsilon>0$ and $\sigma \geq 1+\varepsilon$. For any $a>0$, there exists a constant $C$ (depending only on $a, \varepsilon$, and $\sigma$ ) such that

$$
\left\|g-p s_{N}(g)\right\|_{0, a} \leq C N^{(1+\varepsilon-\sigma) / 2}\|g\|_{\sigma, a}, \quad \forall g \in H_{w_{a}}^{\sigma}(\mathbb{R}) .
$$

Proof. For $g \in L_{w_{a}}^{2}(\mathbb{R})$ denote by $\operatorname{pr}_{N}(g)$ the orthogonal projection of $g$ onto $X_{N+1}$, i.e.,

$$
p r_{N}(g):=\sum_{1 \leq i \leq N+1}\left(g \mid \varphi_{i}\right)_{0, a} \varphi_{i}
$$

where $\left(\varphi_{i}\right)_{i \geq 1}$ is the sequence of the eigenfunctions of $L_{a}$. We have

$$
\left\|g-p s_{N}(g)\right\|_{0, a} \leq\left\|g-p r_{N}(g)\right\|_{0, a}+\left\|p r_{N}(g)-p s_{N}(g)\right\|_{0, a} .
$$

But $p s_{N}\left(p r_{N}(g)\right)=p r_{N}(g)$, and using (3.5), (3.4), we may write (note that the function $\left|w_{a} p s_{N}\left(p r_{N}(g)-g\right)\right|^{2}$ is a polynomial of degree $\left.2 N\right)$

$$
\begin{aligned}
\left\|p r_{N}(g)-p s_{N}(g)\right\|_{0, a}^{2} & =\left\|p s_{N}\left(p r_{N}(g)-g\right)\right\|_{0, a}^{2} \\
& =\int_{-\infty}^{\infty} e^{-y^{2} / 4 a^{2}}\left|\sum_{j=1}^{N+1} e^{y_{j}^{2} / 4 a^{2}}\left(p r_{N}(g)-g\right)\left(y_{j}\right) \ell_{j}(y)\right|^{2} d y \\
& =\sum_{k=1}^{N+1} \omega_{k}\left|\sum_{j=1}^{N+1} \exp \left(\frac{y_{j}^{2}}{4 a^{2}}\right)\left(p r_{N}(g)-g\right)\left(y_{j}\right) \ell_{j}\left(y_{k}\right)\right|^{2},
\end{aligned}
$$

hence by (3.7),

$$
\begin{aligned}
& \left\|p r_{N}(g)-p s_{N}(g)\right\|_{0, a}^{2}=\sum_{k=1}^{N+1} \omega_{k}\left|\exp \left(\frac{y_{k}^{2}}{4 a^{2}}\right)\left(p r_{N}(g)-g\right)\left(y_{k}\right)\right|^{2} \\
& \leq\left\|\rho^{(1+\varepsilon) / 2} w_{a}^{1 / 2}\left(p r_{N}(g)-g\right)\right\|_{\infty}^{2} \sum_{k=1}^{N+1} \omega_{k} \exp \left(\frac{y_{k}^{2}}{4 a^{2}}\right) \rho\left(y_{k}\right)^{-(1+\varepsilon)} \\
& \leq C\left\|p r_{N}(g)-g\right\|_{1+\varepsilon, a}^{2} \sum_{k=1}^{N+1} \omega_{k} \exp \left(\frac{y_{k}^{2}}{4 a^{2}}\right) \rho\left(y_{k}\right)^{-(1+\varepsilon)}
\end{aligned}
$$


(we use here Corollary 3.4 with $\mu=(1+\varepsilon) / 2$ and $\sigma=1+\varepsilon)$. Now following J. V. Uspensky [15] and using (3.4), we have

$$
\lim _{N \rightarrow \infty} \sum_{k=1}^{N+1} \omega_{k} \exp \left(\frac{y_{k}^{2}}{4 a^{2}}\right) \rho\left(y_{k}\right)^{-(1+\varepsilon)}=\int_{-\infty}^{\infty} \rho(y)^{-(1+\varepsilon)} d y<\infty .
$$

Therefore, combining (3.8) and (3.9), we have

$$
\left\|g-p s_{N}(g)\right\|_{0, a} \leq C\left\|g-p r_{N}(g)\right\|_{1+\varepsilon, a} \leq C \lambda_{N+1}^{(1+\varepsilon-\sigma) / 2}\|g\|_{\sigma, a}
$$

and $\lambda_{N+1}=(N+1) / 2$.

We are now ready to give the rate of convergence.

Theorem 3.6. Let $g \in H_{w_{a}}^{\sigma}(\mathbb{R})$ be given with $\sigma \geq 1+\varepsilon$ (and $\varepsilon>0$ ). If $v$ and $v_{N}$ are given by (3.1) and (3.2), then there is a constant $C$ independent of $N>1$ such that

$$
\left\|v-v_{N}\right\|_{2, a} \leq C N^{(1+\varepsilon-\sigma) / 2}\|g\|_{\sigma, a} .
$$

Proof. By Lemma 2.1 and Banach's closed range theorem, $L_{a}: H_{w_{a}}^{2}(\mathbb{R}) \rightarrow L_{w_{a}}^{2}(\mathbb{R})$ is an isomorphism. On the other hand, we have

$$
L_{a}\left(v-v_{N}\right)=g-\tilde{g}_{N}=g-p s_{N}(g) \text {. }
$$

Therefore, there exists $C>0$ such that

$$
\left\|v-v_{N}\right\|_{2, a} \leq C\left\|g-p s_{N}(g)\right\|_{0, a} .
$$

The proof is concluded in view of Theorem 3.5.

Remark 3.7. As a corollary of Theorem 3.6, we can obtain an $L^{\infty}$ estimate of the error on $\left(v-v_{N}\right)$, since we have the inequality

$$
\sup _{y \in \mathbb{R}}\left|h(y) \exp \left(\frac{y^{2}}{8 a^{2}}\right)\right| \leq C\|h\|_{1, a}, \quad \forall h \in H_{w_{a}}^{1}(\mathbb{R}) .
$$

For the case of dimension $d \geq 2$, we consider the elliptic problem

$$
\left\{\begin{array}{l}
\lambda v+L_{a} v=g \quad \text { in } \mathbb{R}^{d}, \\
v \in H_{w_{a}}^{\sigma}\left(\mathbb{R}^{d}\right),
\end{array}\right.
$$

where $g \in H_{w_{a}}^{\sigma}\left(\mathbb{R}^{d}\right)$ is given and $\sigma>d / 2$. The pseudospectral approximation to $(3.11)$ is

$$
\left\{\begin{array}{l}
\lambda v_{N}+L_{a} v_{N}=\tilde{g}_{N} \\
v_{N} \in X_{N+1}
\end{array}\right.
$$

where $X_{N+1}$ is defined in (2.16), $\operatorname{dim} X_{N+1}=(N+1)^{d}$, and

$$
\left\{\begin{array}{l}
\tilde{g}_{N}:=p s_{N}(g)=Q_{N} / w_{a} \in X_{N+1}, \\
Q_{N} \text { is a polynomial whose degree in each variable is } \leq N, \\
Q_{n}=w_{a}\left(y_{\alpha}\right) g\left(y_{\alpha}\right), \quad \alpha \in\{1, \ldots, N+1\}^{d}
\end{array}\right.
$$

(we denote $y_{\alpha}:=\left(y_{\alpha_{1}}, \ldots, y_{\alpha_{d}}\right), y_{k}:=y_{k, N+1, a}$ for $\left.1 \leq k \leq N+1\right)$. 
We can state the following convergence theorem:

Theorem 3.8. Let $\varepsilon>0$ and $\sigma \geq d+\varepsilon$. There is a constant $C>0$ such that if $g \in H_{w_{a}}^{\sigma}\left(\mathbb{R}^{d}\right)$ and $v, v_{N}$ are solutions to (3.11), (3.12) for any $N \geq 1$, one has

$$
\left\|v-v_{N}\right\|_{2, a} \leq C N^{(d+\varepsilon-\sigma) / 2}\|g\|_{\sigma, a} .
$$

Proof. We have $\left\|v-v_{N}\right\|_{2, a} \leq C\left\|g-p s_{N}(g)\right\|_{0, a}$. The conclusion follows, using the analogue of Theorem 3.5 in $\mathbb{R}^{d}$, namely:

Theorem 3.9. Let $\varepsilon>0$ and $\sigma \geq d+\varepsilon$. For any $a>0$, there exists a constant $C$ (depending only on $a, \varepsilon$, and $\sigma$ ) such that

$$
\left\|g-p s_{N}(g)\right\|_{0, a} \leq C N^{(d+\varepsilon-\sigma) / 2}\|g\|_{\sigma, a}, \quad \forall g \in H_{w_{a}}^{\sigma}\left(\mathbb{R}^{d}\right) .
$$

We skip the proof of this last theorem, since it is an easy adaptation of the one given above for Theorem 3.5.

\section{APPROXIMATION IN SOME OTHER UNBOUNDED DOMAINS}

For domains which are Cartesian products of intervals (possibly unbounded) one can use appropriate pseudospectral approximation on each interval. For instance, one can combine Chebyshev or Legendre approximation (cf. [2]) on finite intervals and Hermite approximation on unbounded intervals. In this section we detail two examples: the first for the domain $\Omega=(-1,+1) \times \mathbb{R}$, the second for the case $\Omega=(-\infty, 0)$.

We begin with

$$
\begin{cases}\lambda v+L_{a} v=g & \text { in }(-1,+1) \times \mathbb{R} \\ v( \pm 1, \eta)=0, & \eta \in \mathbb{R}\end{cases}
$$

and $y:=(\xi, \eta) \in(-1,+1) \times \mathbb{R}$. Let

$$
v^{*}(\xi, \eta):=v(\xi, \eta) \exp \left(\frac{|\eta|^{2}}{4 a^{2}}\right), \quad g^{*}(\xi, \eta)=g(\xi, \eta) \exp \left(\frac{|\eta|^{2}}{4 a^{2}}\right) .
$$

Then $v^{*}$ satisfies

$$
\left\{\begin{array}{l}
\lambda v^{*}-a^{2} \Delta v^{*}-\frac{1}{2} \xi \partial_{1} v^{*}+\frac{1}{2} \eta \partial_{2} v^{*}+\frac{1}{2} v^{*}=g^{*} \\
v^{*}( \pm 1, \eta)=0, \quad \eta \in \mathbb{R}
\end{array}\right.
$$

Now we approximate $v^{*}$ and $g^{*}$ by collocation at the Chebyshev nodes in the variable $\xi$ and at the Hermite nodes $y_{k, M+1, a}$ in the variable $\eta$. More precisely, let

$$
\xi_{i}:=\xi_{i, N}:=\cos \frac{i \pi}{N}, \quad 0 \leq i \leq N,
$$

be the Chebyshev Gauss-Lobatto nodes. Denoting by $T_{N}$ the $N$ th Chebyshev polynomial, we have $T_{N}^{\prime}\left(\xi_{i, N}\right)=0,1 \leq i \leq N-1$, and

$$
\int_{-1}^{1} Q(\xi) \frac{d \xi}{\sqrt{1-\xi^{2}}}=\frac{\pi}{N} \sum_{i=1}^{N-1} Q\left(\xi_{i, N}\right)+\frac{\pi}{2 N}(Q(1)+Q(-1))=: \frac{\pi}{N} \sum_{i=0}^{N}{ }^{\prime \prime} Q\left(\xi_{i, N}\right)
$$

for any polynomial $Q$ of degree $\leq 2 N-1$. 
APPROXIMATION BY HERMITE FUNCTIONS

611

Denote by $P_{N, M}$ a polynomial of degree at most $N$ in $\xi$ and of degree at most $M$ in $\eta$. We seek such a polynomial satisfying

$$
\left\{\begin{array}{l}
\left(\lambda P_{N, M}-a^{2} \Delta P_{N, M}-\frac{1}{2} \xi \partial_{1} P_{N, M}+\frac{1}{2} \eta \partial_{2} P_{N, M}+\frac{1}{2} P_{N, M}\right)\left(\xi_{i}, y_{k}\right) \\
=g^{*}\left(\xi_{i}, y_{k}\right),
\end{array}\right.
$$

A proof of convergence can be given for this collocation procedure. We prove first:

Proposition 4.1. There exist $C_{1}, C_{2}>0$ and $N_{0} \geq 1$ such that, for any $N \geq N_{0}$ and polynomial $q$ (in one variable) satisfying $q(+1)=q(-1)=0$ and $\operatorname{deg} q \leq$ $N$, we have

$$
\begin{aligned}
C_{1} \int_{-1}^{+1}\left|q_{\xi}\right|^{2} \frac{d \xi}{\sqrt{1-\xi^{2}}} & \leq \frac{\pi}{N} \sum_{i=0}^{N} \prime\left(\left(-q_{\xi \xi}-\frac{\xi q_{\xi}}{2}\right) q\right)\left(\xi_{i}\right) \\
& \leq C_{2} \int_{-1}^{+1}\left|q_{\xi}\right|^{2} \frac{d \xi}{\sqrt{1-\xi^{2}}} .
\end{aligned}
$$

Proof. We write $q=: \alpha_{N} T_{N}+q_{0}$, where $\operatorname{deg} q_{0} \leq N-1$. Then using (4.3),

$$
-\frac{\pi}{N} \sum_{i=0}^{N}\left(q_{\xi \xi}\left(\xi_{i}\right) q\left(\xi_{i}\right)\right)=\int_{-1}^{+1}-q_{\xi \xi}(\xi) q(\xi) \frac{d \xi}{\sqrt{1-\xi^{2}}},
$$

and it is well known (cf., e.g., [2]) that the right-hand side is uniformly equivalent to

$$
\int_{-1}^{+1}\left|q_{\xi}\right|^{2} \frac{d \xi}{\sqrt{1-\xi^{2}}}
$$

On the other hand,

$$
\begin{aligned}
\frac{\pi}{N} \sum_{i=0}^{N} \xi_{i} q_{\xi}\left(\xi_{i}\right) q\left(\xi_{i}\right)= & \frac{\pi}{N} \alpha_{N}^{2} \sum_{i=0}^{N} \xi_{i} T_{N}^{\prime}\left(\xi_{i}\right) T_{N}\left(\xi_{i}\right) \\
& +\alpha_{N} \int_{-1}^{+1}\left(T_{N} q_{0}^{\prime}+T_{N}^{\prime} q_{0}\right) \frac{\xi d \xi}{\sqrt{1-\xi^{2}}} \\
& +\int_{-1}^{+1} q_{0}^{\prime} q_{0} \frac{\xi d \xi}{\sqrt{1-\xi^{2}}} \\
= & \int_{-1}^{+1} \xi q_{\xi} q \frac{d \xi}{\sqrt{1-\xi^{2}}}+\alpha_{N}^{2} \frac{\pi N}{2},
\end{aligned}
$$

where we use the fact that

$$
\frac{\pi}{N} \sum_{i=0}^{N}{ }^{\prime \prime} \xi_{i} T_{N}^{\prime}\left(\xi_{i}\right) T_{N}\left(\xi_{i}\right)=2 \int_{-1}^{+1} T_{N}^{\prime} T_{N} \frac{\xi d \xi}{\sqrt{1-\xi^{2}}}=\pi N
$$


Now,

$$
0 \leq \frac{1}{2} \int_{-1}^{1} q^{2} \frac{d \xi}{\left(1-\xi^{2}\right)^{3 / 2}}=-\int_{-1}^{1} \xi q_{\xi} q \frac{d \xi}{\sqrt{1-\xi^{2}}} \leq C \int_{-1}^{1} q_{\xi}^{2} \frac{d \xi}{\sqrt{1-\xi^{2}}}
$$

while the remaining term on the right of (4.7) can be bounded as follows:

$$
\frac{\pi N}{2} \alpha_{N}^{2} \leq \frac{C}{N} \int_{-1}^{1} q_{\xi}^{2} \frac{d \xi}{\sqrt{1-\xi^{2}}}
$$

Therefore, for $N$ large enough, we obtain (4.5).

By the previous proposition and the results in $\S 3$ it is straightforward to prove the next theorem.

Theorem 4.2. There exist $C_{1}, C_{2}>0$ such that, for any polynomial $p_{N, M}$ of degree $N$ in the variable $\xi(N$ sufficiently large $)$ and of degree $M$ in the variable $\eta$, we have

$$
\begin{aligned}
& C_{1} \int_{(-1,1) \times R}\left|\nabla p_{N, M}\right|^{2} \frac{d \xi d \eta}{\sqrt{1-\xi^{2}} w_{a}(\eta)} \\
& \leq \frac{\pi}{N} \sum_{i=0}^{N} \sum_{k=1}^{M}\left[\left(-a^{2} \Delta p_{N, M}-\frac{1}{2} \xi \partial_{1} p_{N, M}+\frac{1}{2} \eta \partial_{2} p_{N, M}+\frac{1}{2} p_{N, M}\right)\right. \\
& \left.\quad \times\left(\xi_{i}, y_{k}\right) p_{N, M}\left(\xi_{i}, y_{k}\right)\right] \omega_{k} \\
& \leq C_{2} \int_{(-1,1) \times R}\left|\nabla p_{N, M}\right|^{2} \frac{d \xi d \eta}{\sqrt{1-\xi^{2}} w_{a}(\eta)} .
\end{aligned}
$$

Stability for problem (4.4) is now a direct consequence of the above result. The estimates which show the spectral convergence are easily obtained by separation of variables and then applying Theorem 3.5 and the estimates on the Chebyshev interpolation projectors (cf. [2]).

Next we consider problem (1.1), when $\Omega=(-\infty, 0)$, with the boundary condition $u(t, 0)=0, t>0$. After the substitutions proposed in $\S \S 1$ and 3 the problem becomes

$$
\begin{cases}v_{s}^{*}-v_{y y}^{*}+\frac{1}{2} y v_{y}^{*}+\frac{1}{2} v^{*}=g^{*}, & y<0, s>0, \\ v^{*}(s, 0)=0, & s>0, \\ v^{*}(0, y)=v_{0}^{*}(y), & y \leq 0 .\end{cases}
$$

Suppose now that $g^{*}$ and $v_{0}^{*}$ are the restrictions of certain functions $g$ and $v_{0}$ defined in $\mathbb{R}$. Then $v^{*}$ can be considered as the restriction of the function $\hat{v}: \mathbb{R} \rightarrow \mathbb{R}$ which solves the problem

$$
\begin{cases}\hat{v}_{s}-\hat{v}_{y y}+\frac{1}{2} y \hat{v}+\frac{1}{2} \hat{v}=\hat{g}, & y \in \mathbb{R}-\{0\}, s>0 \\ \hat{v}(s, 0)=0, & s>0, \\ \hat{v}(0, y)=\hat{v}_{0}(y), & y \in \mathbb{R} .\end{cases}
$$


For a given function $\varphi$, we denote by $\varphi^{+}$and $\varphi^{-}$respectively the even and the odd part of $\varphi$. As the space of odd (resp. even) functions is stable under the action of the operator $\varphi \mapsto-\varphi_{y y}+\frac{y}{2} \varphi_{y}$, it is easy to check that the solution $\hat{v}=\hat{v}^{+}+\hat{v}^{-}$of problem (4.10) can be obtained by solving

$$
\begin{cases}\hat{v}_{s}^{ \pm}-\hat{v}_{y y}^{ \pm}+\frac{1}{2} y \hat{v}_{y}^{ \pm}+\frac{1}{2} \hat{v}^{ \pm}=\hat{g}^{ \pm}, & y \in \mathbb{R}-\{0\}, s>0, \\ \hat{v}^{ \pm}(s, 0)=0, & s>0, \\ \hat{v}^{ \pm}(0, y)=\hat{v}_{0}^{ \pm}(y), & y \in \mathbb{R},\end{cases}
$$

according to the determination of the even or the odd part of $v$.

We want to approximate the solution $\hat{v}^{+}$of (4.11) (similarly we shall argue for $\hat{v}^{-}$). For this purpose, consider the nodes $y_{j, N+1, a}, j=1, \ldots, 2 N+1$. We have $y_{N+1,2 N+1, a}=0$ and $y_{j, 2 N+1, a}=-y_{2 N+2-j, 2 N+1, a}, j=1, \ldots, N$. Denote by $X_{N}^{+}$the space of the even polynomials $p_{N}^{+}$of degree at most $2 N$ satisfying $p_{N}^{+}(0)=0$. One has $\operatorname{dim}\left(X_{N}^{+}\right)=N$.

Therefore, an element of $X_{N}^{+}$is uniquely determined by its values at the nodes $y_{j, 2 N+1, a}, j=1, \ldots, N$. Then we approximate $\hat{v}^{+}$by a polynomial $p_{N}^{+} \in X_{N}^{+}$such that

$$
\left\{\begin{aligned}
\left(p_{N, s}^{+}-p_{N, y y}^{+}+\frac{1}{2} y p_{N, y}^{+}+\frac{1}{2} p_{N}^{+}\right)\left(y_{j, N+1, a}\right)=\hat{g}^{+}\left(y_{j, 2 N+1, a}\right), & \\
& j=1, \ldots, N, s>0, \\
p_{N}^{+}\left(0, y_{j, 2 N+1, a}\right)=\hat{v}_{0}^{+}\left(y_{j, 2 N+1, a}\right), \quad j= & 1, \ldots, N .
\end{aligned}\right.
$$

Using the fact that $p_{N}^{+}$is even, the equations in (4.12) also hold for the negative nodes. An analysis of stability and convergence for (4.12) can easily be carried out. In particular, by the theory developed in $\S 3$, for the steady state version of (4.11) and (4.12) we obtain the estimate

$$
\left\|\left(\hat{v}^{+}-p_{N}^{+}\right) w_{a}^{1 / 2}\right\|_{H^{1}((-\infty, 0))} \leq C(2 N)^{(1+\varepsilon-\sigma) / 2}\|\hat{g}\|_{H_{w_{a}}^{o}(\mathbb{R})}, \quad \sigma>1+\varepsilon .
$$

A similar technique is used to approximate the solution $\hat{v}^{-}$in (4.11). This time, $X_{N}^{-}$will denote the space of odd polynomials $p_{N}^{-}$of degree at most $2 N-1$ (thus satisfying $\left.p_{N}^{-}(0)=0\right)$. Again, we have $\operatorname{dim}\left(X_{N}^{-}\right)=N$, and the corresponding collocation scheme is obtained by writing $p_{N}^{-}, \hat{g}^{-}$, and $\hat{v}_{0}^{-}$in place of $p_{N}^{+}, \hat{g}^{+}$, and $\hat{v}_{0}^{+}$in (4.12). The approximation of $v^{*}$ in (4.9) is $p_{N}^{+}+p_{N}^{-}$restricted to $(-\infty, 0)$.

This kind of approximation involves the resolution of two different $N \times N$ differential systems. On the other hand, $N$ does not need to be very large in application since, as shown by (4.13), the rate of convergence is in general very high.

An approximation of $v^{*}$ in (4.9) can also be given in the following way. We write again equation (4.10), but this time $\hat{g}$ is such that $\hat{g}(y)=g^{*}(y)$ if $y>0$ and $\hat{g}(y)=g^{*}(-y)$ if $y<0$. Therefore, the corresponding solution $p_{N}^{-}$to (4.12) is already an approximation of $\hat{v}$ without evaluating $p_{N}^{-}$. In this case we 
only solve an $N \times N$ differential system. Nevertheless, the last procedure yields results that are not very good in general. This can be explained by noting that, even if $g^{*}$ is a very regular function, its even continuation can be an irregular function. This may affect very badly the convergence, as observed by numerical experiments. This difficulty may be avoided by using the first approach.

For the reader's convenience we give the expressions of the entries of the matrices associated with the discretization in (4.12). The Lagrange interpolation basis in $X_{N}^{+}$is the following (here, for simplicity, we take $a:=1$ ):

$$
\ell_{j}^{+}(y)=\frac{2 y h_{2 N+1}(y)}{h_{2 N+1}^{\prime}\left(y_{j}\right)\left(y^{2}-y_{j}^{2}\right)}, \quad y_{j}=y_{j, 2 N+1, a}, \quad j=1, \ldots, N .
$$

Then we get

$$
\begin{gathered}
\left(\ell_{j, y}^{+}\right)\left(y_{i}\right)= \begin{cases}\frac{2 y_{i} h_{2 N+1}^{\prime}\left(y_{i}\right)}{h_{2 N+1}^{\prime}\left(y_{i}\right)\left(y_{i}^{2}-y_{j}^{2}\right)} & \text { if } i \neq j, \\
\frac{y_{j}^{2}+2}{4 y_{j}} & \text { if } i=j,\end{cases} \\
\left(-\ell_{j, y y}^{+}+\frac{y}{2} \ell_{j, y}^{+}\right)\left(y_{i}\right)= \begin{cases}\frac{4 h_{2 N+1}^{\prime}\left(y_{i}\right)\left(y_{i}^{2}+y_{j}^{2}\right)}{h_{2 N+1}^{\prime}\left(y_{j}\right)\left(y_{i}^{2}-y_{j}^{2}\right)^{2}} & \text { if } i \neq j, \\
-\frac{1}{12}\left(y_{j}^{2}-4 N\right)+\frac{1}{8 y_{j}^{2}}\left(y_{j}^{2}+2\right)^{2} & \text { if } i=j .\end{cases}
\end{gathered}
$$

Similarly, in $X_{N}^{-}$one has

$$
\begin{gathered}
\ell_{j}^{-}(y)=\frac{2 y_{j} h_{2 N+1}(y)}{h_{2 N+1}^{\prime}\left(y_{j}\right)\left(y^{2}-y_{j}^{2}\right)}, \quad j=1, \ldots, N, \\
\left(\ell_{j, y}^{-}\right)\left(y_{i}\right)= \begin{cases}\frac{2 y_{j} h_{2 N+1}^{\prime}\left(y_{i}\right)}{h_{2 N+1}^{\prime}\left(y_{j}\right)\left(y_{i}^{2}-y_{j}^{2}\right)} & \text { if } i \neq j, \\
\frac{y_{j}^{2}-2}{4 y_{j}} & \text { if } i=j,\end{cases} \\
\left(-\ell_{j, y y}^{-}+\frac{y}{2} \ell_{j, y}^{-}\right)\left(y_{i}\right)= \begin{cases}\frac{8 y_{i} y_{j} h_{2 N+1}^{\prime}\left(y_{i}\right)}{h_{2 N+1}^{\prime}\left(y_{j}\right)\left(y_{i}^{2}-y_{j}^{2}\right)^{2}} \\
-\frac{1}{12}\left(y_{j}^{2}-4 N\right)+\frac{1}{8 y_{j}^{2}}\left(y_{j}^{4}-4\right)+\frac{1}{2} & \text { if } i=j .\end{cases}
\end{gathered}
$$

\section{Numerical tests}

We devote this section to the discussion of several numerical experiments. We begin with some examples with $d=1$. Consider the problem

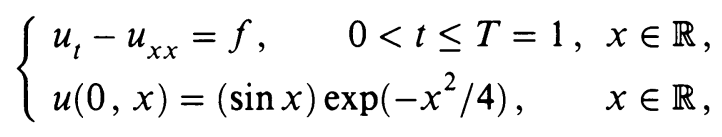


where $f$ is chosen such that the solution is

$$
u(x, t)=\frac{\sin x}{\sqrt{1+t}} \exp \left(\frac{-x^{2}}{4(1+t)}\right) .
$$

After the transformation suggested in $\S 1$ (then putting $v^{*}(s, y):=$ $\left.v(s, y) \exp \left(y^{2} / 4\right)\right)$, equation (5.1) becomes (here $\left.S:=\log (2)\right)$

$$
\begin{cases}v_{s}^{*}-v_{y y}^{*}+\frac{1}{2} y v_{y}^{*}+\frac{1}{2} v^{*}=y \cos \left(y e^{s / 2}\right)+e^{s / 2} \sin \left(y e^{s / 2}\right), & y \in \mathbb{R}, 0<s \leq S, \\ v^{*}(0, y)=\sin y, \quad y \in \mathbb{R} . & \end{cases}
$$

The solution of (5.2) is approximated by $p_{N}$ as in (3.6) $(a=1)$. Let

$$
E_{N}=\left\|\left(v^{*}-p_{N}\right) w_{1}^{-1 / 2}\right\|_{L^{2}(\mathbb{R})},
$$

when $v^{*}$ and $p_{N}$ are evaluated at the time $S=\log (2)$. Since we are mainly concerned in checking the accuracy of pseudospectral approximation studied in $\S \S 3$ and 4, we do not look for an optimal discretization in time. Therefore, we used explicit first-order forward differencing to advance in time. Denoting by $N_{\text {IT }}$ the number of iterations (the time step therefore is $S / N_{\text {IT }}$ ), Table 5.1 shows the error $E_{20}$ versus $N_{\mathrm{IT}}$.

TABLE 5.1

Error $E_{20}$ for different values of $N_{\mathrm{IT}}$

\begin{tabular}{|r|c|}
\hline$N_{\text {IT }}$ & $E_{20}$ \\
\hline 250 & $.2487 \mathrm{E}-02$ \\
1000 & $.6203 \mathrm{E}-03$ \\
4000 & $.1550 \mathrm{E}-03$ \\
16000 & $.3886 \mathrm{E}-04$ \\
\hline
\end{tabular}

We chose large values of $N_{\text {IT }}$ in order to emphasize the error with respect to the spatial variable.

We recall that the maximum size of the time step in order to get stability is given by $2 /(N+1)$. Actually, this can be easily determined by knowing the eigenvalues of the matrix relative to the spatial discretization (see Remark 3.1).

To better examine the convergence behavior with respect to $N$, we consider the elliptic problem

$$
-v_{y y}^{*}+\frac{1}{2} y v_{y}^{*}+\frac{1}{2} v^{*}=\frac{1}{2}(y-1) e^{y}, \quad y \in \mathbb{R} .
$$

This admits the solution $v^{*}(y)=e^{y}$. The approximate solution $p_{N}$ (given by (3.6)) is obtained by solving the corresponding linear system by Gaussian 
TABLE 5.2

Errors versus $N$ for the approximation of problem (5.3)

\begin{tabular}{|c|c|c|c|}
\hline$N+1$ & $\left\|\left(v^{*}-p_{N}\right) w_{1}^{1 / 2}\right\|_{L^{2}(\mathbb{R})}$ & $E_{N}^{\infty}$ & $\left\|\left(v^{*}-p_{N}\right)_{y} w_{1}^{1 / 2}\right\|_{L^{2}(\mathbb{R})}$ \\
\hline 8 & $.5389 \mathrm{E}-01$ & $.3004 \mathrm{E}-01$ & $.7416 \mathrm{E}-00$ \\
16 & $.1392 \mathrm{E}-04$ & $.5985 \mathrm{E}-05$ & $.7674 \mathrm{E}-03$ \\
24 & $.6959 \mathrm{E}-09$ & $.2415 \mathrm{E}-09$ & $.8875 \mathrm{E}-07$ \\
\hline
\end{tabular}

elimination. Let $E_{N}^{\infty}=\max _{1 \leq j \leq N+1}\left\{\left|v^{*}-p_{N}\right|\left(y_{j}\right) \exp \left(-y_{j}^{2} / 8\right)\right\}$ (see Remark 3.7); then, in Table 5.2 we report the error for different norms. It is clear that we have convergence of spectral type.

Similar results can be obtained for $d=2$. We give an example where different techniques are used to approximate the spatial operator in each direction (see $\S 4)$. On the domain $(-1,1) \times \mathbb{R}$, we solve the equation (4.2) when $a=1$, $\lambda=0$, and $g^{*}$ is such that $v^{*}(\xi, \eta)=\sin \left(\eta\left(1-\xi^{2}\right)\right)$. The approximation is performed as in (4.4). In Table 5.3, we give the error

$$
E_{N, M}=\left(\frac{\pi}{N} \sum_{i=0}^{N} \sum_{k=1}^{M}\left(v^{*}-p_{N, M}\right)^{2}\left(\xi_{i}, y_{k}\right) \omega_{k}\right)^{1 / 2}
$$

for various $N$ and $M$. We note that in the relevant system the number of unknowns is $(N-1) \times(M+1)$.

TABLE 5.3

Error for the Chebyshev-Hermite approximation to (4.2)

\begin{tabular}{|r|r|c|}
\hline$N$ & $M$ & $E_{N, M}$ \\
\hline 4 & 4 & $.6025 \mathrm{E}-00$ \\
8 & 8 & $.7399 \mathrm{E}-02$ \\
12 & 12 & $.1338 \mathrm{E}-03$ \\
4 & 8 & $.5218 \mathrm{E}-00$ \\
8 & 4 & $.3184 \mathrm{E}-01$ \\
12 & 6 & $.5098 \mathrm{E}-02$ \\
6 & 12 & $.9172 \mathrm{E}-01$ \\
\hline
\end{tabular}


Next we analyze an equation on the half-plane,

$$
\begin{cases}-\Delta v^{*}+\frac{1}{2} y \nabla v^{*}+v^{*}=g^{*} & \text { for } y=(\xi, \eta) \in \mathbb{R} \times(-\infty, 0), \\ v(\xi, 0)=0, & \xi \in \mathbb{R} .\end{cases}
$$

The function $g^{*}$ is chosen such that $v^{*}(\xi, \eta)=\eta+e^{\xi} \sin \eta$, which is an odd function in the variable $\eta$. The approximation is performed by collocation at the $N+1$ Hermite nodes in $\mathbb{R}$ for the variable $\xi$ and the $M$ nodes $y_{j, 2 M+1,1}$, $j=1, \ldots, M$, for the variable $\eta$. The approximation, which is a polynomial of degree at most $N$ in the variable $\xi$ and a polynomial of $X_{M}^{-}$in the variable $\eta$, is denoted by $p_{N, M}$. Let

$$
E_{N, M}=\left(\sum_{i=1}^{N+1} \sum_{j=1}^{M}\left(v^{*}-p_{N, M}\right)^{2}\left(y_{i, N+1,1}, y_{j, 2 M+1,1}\right) \omega_{i, N+1,1} \omega_{j, 2 M+1,1}\right)^{1 / 2} \text {; }
$$

then, in Table 5.4, $E_{N, M}$ is reported for some values of $N$ and $M$ (that are chosen in order to have the same number of nodes in each direction). The system is solved by Gaussian elimination.

TABLE 5.4

Errors for the approximation on the half-plane

\begin{tabular}{|r|r|l|}
\hline \multicolumn{1}{|r|}{$N$} & $M$ & \multicolumn{1}{|c|}{$E_{N, M}$} \\
\hline 4 & 5 & 2.6395 \\
6 & 7 & 0.6129 \\
8 & 9 & 0.1110 \\
10 & 11 & $0.1675 \mathrm{E}-01$ \\
12 & 13 & $0.2175 \mathrm{E}-02$ \\
\hline
\end{tabular}

Finally, we end the section with the following problem:

$$
\begin{cases}-\Delta v^{*}-\frac{1}{2} \xi \partial_{1} v^{*}+\frac{1}{2} \eta \partial_{2} v^{*}+\frac{1}{2} v^{*}=g^{*} & \text { in }(-1,1) \times(-\infty, 0), \\ v^{*}(\xi, 0)=0, & \xi \in[-1,1], \\ v^{*}( \pm 1, \eta)=0, & \eta \leq 0,\end{cases}
$$

where $g^{*}$ is such that $v^{*}(\xi, \eta)=\eta^{2} \cos \frac{\pi}{2} \xi+\left(1-\xi^{2}\right)(\cos \eta-1)$, which is an even function in the variable $\eta$. As usual, by $p_{N, M}$ (polynomial of degree at most $N$ in the variable $\xi$ and polynomial in $X_{M}^{+}$for the variable $\eta$ ) we denote the approximate solution obtained combining Chebyshev collocation with Hermite collocation as in $\S 4$. Table 5.5 shows the error

$$
E_{N, M}=\left(\frac{\pi}{N} \sum_{i=0}^{N} \sum_{k=1}^{M}\left(v^{*}-p_{N, M}\right)^{2}\left(\xi_{i, N}, y_{k, 2 M+1,1}\right) \omega_{k, 2 M+1,1}\right)^{1 / 2} .
$$


TABLE 5.5

Errors for the approximation of (5.4)

\begin{tabular}{|r|r|c|}
\hline \multicolumn{1}{r|}{$N$} & $M$ & $E_{N, M}$ \\
\hline 4 & 3 & $.7686 \mathrm{E}-02$ \\
6 & 5 & $.2057 \mathrm{E}-03$ \\
8 & 7 & $.3065 \mathrm{E}-05$ \\
10 & 9 & $.2966 \mathrm{E}-07$ \\
12 & 11 & $.1998 \mathrm{E}-09$ \\
14 & 13 & $.1014 \mathrm{E}-11$ \\
\hline
\end{tabular}

As before, $N$ and $M$ are such that the system has the same number of unknowns in each variable.

Remark 5.1. We should point out that, as is usual in pseudospectral approximation, when other parabolic or elliptic operators are involved, one can use the same basis of Hermite functions. For instance, consider the following elliptic equation:

$$
-\Delta u+b \cdot \nabla u+\lambda u=f \quad \text { in } \mathbb{R}^{d},
$$

where $\lambda>0, b \in \mathbb{R}^{d}$, and $f$ decays at infinity at a suitable rate. Then writing

$$
v(x):=e^{x^{2} / 4} u(x), \quad g(x):=e^{x^{2} / 4} f(x),
$$

one checks that $v$ satisfies the new equation

$$
-\Delta v+(x+b) \cdot \nabla v+\left(d / 2+\lambda-\frac{1}{4}|x|^{2}-\frac{1}{2} b \cdot x\right) v=g .
$$

This equation can be solved numerically by the collocation method as explained above. The matrix related to the corresponding linear system is easily obtained by the matrices of first and second derivatives given in $\S 3$.

The numerical approximation of semilinear parabolic or elliptic equations can be treated in a similar way.

Remark 5.2. In the process of computing the nodes, weights, and entries of the derivative matrices, one encounters numerical difficulties due to the behavior of Hermite polynomials of high degree. For a study of this aspect, see [7], where appropriate numerical procedures are suggested.

\section{BIBLIOGRAPHY}

1. C. Canuto, S. I. Hariharan, and L. Lustman, Spectral methods for exterior elliptic problems, ICASE, report \# 21, 1984.

2. C. Canuto, H. Y. Hussaini, A. Quarteroni, and T. A. Zang, Spectral methods in fluid dynamics, Springer Series in Computational Physics, Springer-Verlag, Heidelberg, 1987. 
3. O. Coulaud, D. Funaro, and O. Kavian, Laguerre spectral approximation of elliptic problems in exterior domains, Proc. Internat. Congr. Spectral and High Order Methods (ICOSAHOM) (Como, Italy, 1989), Computer Methods in Applied Mechanics and Engineering \#80 (1990), 451-458 (Elsevier Science Publishers B. V. (North-Holland)).

4. P. J. Davis and P. Rabinowitz, Methods of numerical integration, Academic Press, New York, 1984.

5. M. Escobedo and O. Kavian, Variational problems related to self-similar solutions of the heat equation, Nonlinear Anal. Theory Methods Appl. 11 (1987), 1103-1133.

6. _ Asymptotic behaviour of positive solutions of a non-linear heat equation, Houston J. Math. 13 (1988), 39-50.

7. D. Funaro, Computational aspects of pseudospectral Laguerre approximations, Appl. Numer. Math. (submitted).

8. D. Gottlieb and S. A. Orszag, Numerical analysis of spectral methods: Theory and applications, CBMS Regional Conf. Ser. in Appl. Math., SIAM, Philadelphia, PA, 1977.

9. O. Kavian, Remarks on the large time behaviour of a nonlinear diffusion equation, Ann. Inst. H. Poincaré 4 (1987), 423-452.

10. __ The Navier-Stokes equation in weighted Sobolev spaces, Prépublications de l'Institut Elie Cartan (Nancy, France), 1991.

11. J. Leray, Sur le mouvement d'un liquide visqueux emplissant l'espace, Acta Math. 63 (1934), 193-248.

12. J. L. Lions and E. Magenes, Problèmes aux limites non-homogènes et applications, Vol. 1, Dunod, Paris, 1968.

13. Y. Maday, B. Pernaud-Thomas, and $\mathrm{H}$. Vandeven, Une réhabilitation des méthodes de type Laguerre, Rech. Aérospat. 6 (1985), 353-375.

14. G. Szegö, Orthogonal polynomials, Amer. Math. Soc., Providence, RI, 1959.

15. J. V. Uspensky, On the convergence of quadrature formulas related to an infinite interval, Trans. Amer. Math. Soc. 30 (1928), 542-559.

Dipartimento di Matematica, Università di Pavia, Strada Nuova 65, 27100 Pavia, Italy

Institut Elie Cartan, Departement de Mathematiques, Université de Nancy I, B. P. 239, 54506 Vandeuvre les Nancy Cedex, France 